\title{
Trisomy 15 rescue with jumping translocation of distal 15q in Prader-Willi syndrome
}

Koenraad Devriendt, Paul Petit, Gert Matthijs, Joris R Vermeesch, Maureen Holvoet, Alain De Muelenaere, Peter Marynen, Jean-Jacques Cassiman, Jean-Pierre Fryns
Centre for Human Genetics, University Hospital Leuven, Herestraat 49, B-3000 Leuven, Belgium K Devriendt P Petit

G Matthijs

J R Vermeesch

$M$ Holvoet

P Marynen

J-J Cassiman

J-P Fryns

MPIOLV ter Engelen, Klerken, Belgium A De Muelenaere

Correspondence to: Dr Devriendt.

Received 5 August 1996 Revised version accepted for publication 13 December 1996

\begin{abstract}
We report a patient with Prader-Willi syndrome (PWS) and mosaicism for a de novo jumping translocation of distal chromosome 15q, resulting in partial trisomy for 15q24-qter. A maternal uniparental heterodisomy for chromosome 15 was present in all cells, defining the molecular basis for the PWS in this patient. The translocated distal $15 \mathrm{q}$ fragment was of paternal origin and was present as a jumping translocation, involving three different translocation partners, chromosomes 14q, 4q, and 16p. The recipient chromosomes appeared cytogenetically intact and interstitial telomere DNA sequences were present at the breakpoint junctions. This strongly suggests that the initial event leading to the translocation of distal 15q was a non-reciprocal translocation, with fusion between the $15 \mathrm{q} 24$ breakpoint and the telomeres of the recipient chromosomes. These observations are best explained by a partial zygotic trisomy rescue and comprise a previously undescribed mechanism leading to partial trisomy.

(F Med Genet 1997;34:395-399)
\end{abstract}

Keywords: Prader-Willi syndrome; jumping translocation; uniparental disomy; partial trisomy

Prader-Willi syndrome (PWS) is caused by the absence of a paternal genetic contribution to chromosomal region 15q11-13, either through deletion on the paternal chromosome or through the presence of maternal uniparental disomy for chromosome 15 (UPD15 mat). ${ }^{12}$ Other chromosomal aberrations have been reported in PWS, such as unbalanced translocations or a marker chromosome derived from chromosome 15 , resulting in either a deletion in paternal chromosome $15 q 11-13$ or in UPD15 mat. ${ }^{3-9}$ Jumping translocations are extremely rare and describe the translocation of the same chromosomal fragment to different translocation partners in different cell lines of a single person. Interestingly, the initial description of a jumping translocation was also in a patient with PWS. ${ }^{10}$ The majority of jumping translocations reported so far involve heterochromatic chromosomal regions, such as telomeres, centromeres, or satellites. ${ }^{11}$ Rarely, jumping translocations with an interstitial breakpoint in one of the chromosomes have been reported and, interestingly, most of these cases were patients with the PWS, with a breakpoint in chromosomal region $15 q 11-13 .^{45}$

We report here a patient with Prader-Willi syndrome carrying a jumping translocation resulting in a partial trisomy.

\section{Subjects and methods}

CLINICAL DATA

The patient is a girl, the third child of healthy, non-consanguineous parents. Family history is negative with regard to mental retardation or congenital malformations. At the time of birth, the mother was 25 and the father 29 years old. During pregnancy, fetal movements were reduced and there was polyhydramnios. She was born at term with a birth weight of $2200 \mathrm{~g}$ ( $3 \mathrm{rd}$ centile $=2500 \mathrm{~g}$ ). The neonatal period and infancy were characterised by severe hypotonia and major feeding difficulties, necessitating frequent hospital admission and nasogastric tube feeding. Weight gain was poor. Around the age of 2 years, she developed a marked change in feeding behaviour, with an increase in appetite. This led to the gradual development of obesity during childhood. At the age of 22 months, weight was $10 \mathrm{~kg}$ (3rd-25th centile), height $85 \mathrm{~cm}$ (50th centile), and head circumference $46 \mathrm{~cm}$ ( $3 \mathrm{rd}-25$ th centile). At the age of 10 years, weight was $42.5 \mathrm{~kg}$ (90th-97th centile), height $135 \mathrm{~cm}$ (25th-50th centile), and head circumference $52 \mathrm{~cm}$ (25th-50th centile). She suffered from recurrent urinary infections and at the age of 2 years unilateral vesicoureteral reflux was surgically corrected. Psychomotor development was severely delayed: she could sit at 2.5 years and walk at 3 years. First words appeared at 8 years. Now, at the age of 22 years, there is truncal obesity, with weight $73.5 \mathrm{~kg}$ (90th-97th centile), height $153 \mathrm{~cm}$ (3rd-25th centile), and head circumference $55 \mathrm{~cm}$ ( 75 th centile). She is dysmorphic, with short hands (hand length $15.5 \mathrm{~cm}$ and finger length $6.5 \mathrm{~cm}$, both below the 3rd centile) and short feet, low set ears, and almond shaped eyes (fig 1). The saliva is sticky and the teeth are carious. There is no hypopigmentation. The corners of the mouth are downturned and the palate is high arched. She is myopic. There is hypotonia, with genu valgum and dorsal kyphosis. She is severely mentally retarded and exhibits obsessive behaviour, temper tantrums, and skin picking. Sleep is disturbed, with frequent awakening. There is primary amenorrhoea. She forages food. Pain sensitivity is diminished. She fulfils all major and seven minor criteria of the 


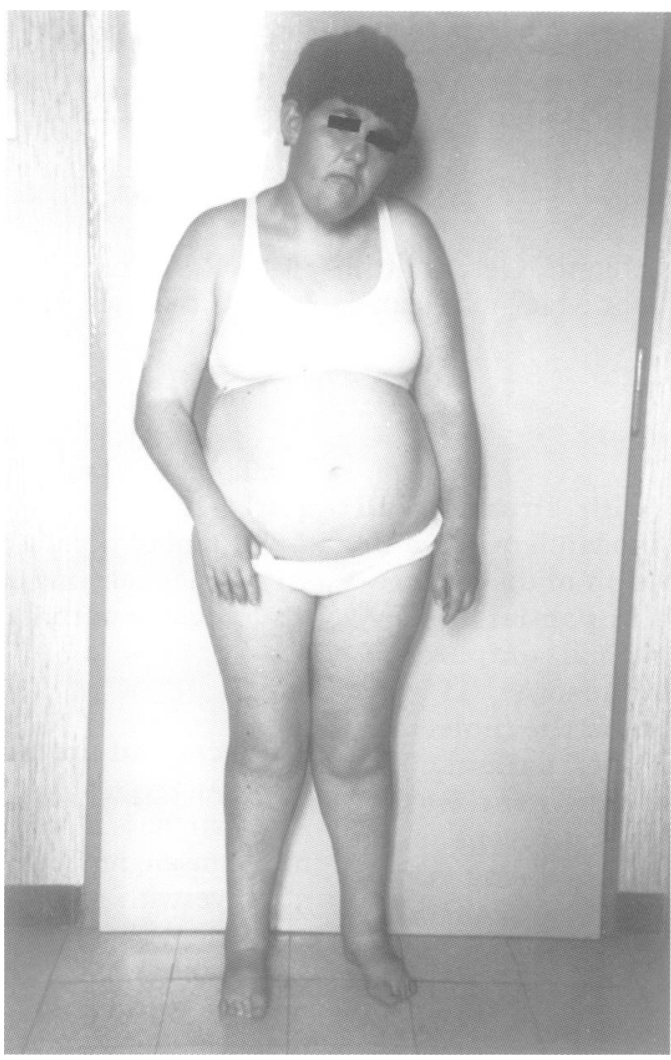

Figure 1 Clinical features of the patient at 22 years. Note the truncal obesity, short stature, small hands and feet, and hypotonia, with downturned corners of the mouth.

recently proposed diagnostic criteria for Prader-Willi syndrome. ${ }^{12}$

\section{METHODS}

Cytogenetic analysis

Chromosome studies on peripheral lymphocytes and skin fibroblasts were performed according to standard cytogenetic techniques, and karyotyping was by Giemsa banding. Fluorescent in situ hybridisation (FISH) and detection were carried out using coatasome 15 chromosome paint and SNRPN/PML probes, obtained from ONCOR (Gaithersburg, MD). The PML probe supplied together with the SNRPN probe is present as a control probe and recognises sequences in 15q22. A biotin labelled FES cosmid probe (15q26.1) (a gift from E Schoenmakers, Leuven) and oligonucleotide (TTAGGG)7 probes were applied as described by Pinkel et $a l^{13}$ and Vermeesch et $a l^{14}$ respectively. Pictures were taken by digital imaging microscopy using a cooled charge coupled device camera system (Photometrics). Merging and pseudocolouring were performed using the SmartCapture software (Vysis, Stuttgart, Germany). At least 30 metaphases were examined with each probe.

Molecular analysis

Genomic DNA was extracted from peripheral white blood cells and cultured fibroblasts and analysed by Southern blotting using probes

Table 1 Distribution of the different karyotypes

\begin{tabular}{llllll}
\hline Karyotype & $46, X X$ & $46, X X, 4 q+$ & $46, X X, 14 q+$ & $46, X X, 16 p+$ & Total \\
\hline Lymphocytes (No of cells) & 8 & 11 & 85 & 3 & 107 \\
Fibroblasts (No of cells) & 51 & 14 & 35 & 0 & 100 \\
\hline
\end{tabular}

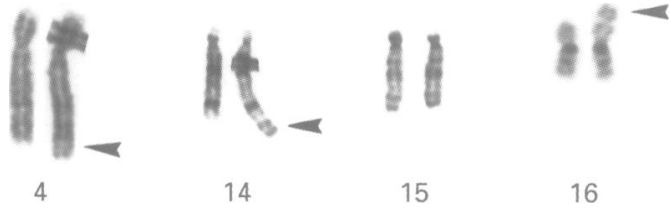

Figure $2 G$ banded partial karyotype showing, from left to right, chromosomes $4,14,15$, and 16. Note the $4 q+$, $14 q+$, and $16 p+$ (arrowheads). This fragment was tentatively identified as 15q24-qter.

PW71 ${ }^{15}$ (a gift from Dr B Horsthemke) and DN34E as previously described. ${ }^{16}$

Analysis of polymorphic microsatellite markers was done using PCR amplification on the patient (white blood cells and fibroblasts) and her mother (white blood cells). DNA from the father was not available. The following loci on chromosome 15 were examined: D15S122, D15S165, APW, D15S123, FES, D15S107, and D15S120. The primer sequences were obtained from GDB.

\section{Results}

CYTOGENETIC ANALYSIS

Cytogenetic analysis on both lymphocyte and fibroblast tissues showed a normal 46,XX cell line and, in addition, unbalanced karyotypes with partial trisomy 15q24-qter (table 1, fig 2). Mosaicism consistent with a jumping translocation was observed with a predominant $46, \mathrm{XX}, 14 \mathrm{q}+$ cell line as well as $46, \mathrm{XX}, 4 \mathrm{q}+$ and $46, \mathrm{XX}, 16 \mathrm{p}+$ cell lines (table 1 , fig 2). Chromosome painting with a chromosome 15 specific probe confirmed that the extra chromosomal material originated from chromosome 15 (fig 3A). FISH using a cosmid probe for the FES gene, located on chromosome $15 \mathrm{q} 26.1$, showed three signals in all unbalanced metaphases, one on the distal region of each chromosome 15 and one on the extra material translocated onto chromosomes $14 \mathrm{q}$, $4 \mathrm{q}$, and $16 \mathrm{p}$ (fig 3B). FISH using the SNRPN/ PML cocktail probe showed normal signals at $15 \mathrm{q} 11$ and $15 \mathrm{q} 22$ on both chromosomes, excluding a deletion involving the SNRPN gene (fig 3C). This also confirmed that the translocated region in the unbalanced cells originated from the distal $15 \mathrm{q}$ region, with a breakpoint located between 15q22 (PMLcontrol probe) and 15q26.1 (FES locus).

The karyotypes of the parents and sibs were normal after $\mathrm{G}$ banding.

PARENTAL ORIGIN OF THE CHROMOSOMES 15 AND TRANSLOCATED FRAGMENT DISTAL 15q The results obtained with probes $\mathrm{DN} 34$ and PW71 showed hypermethylation of the PWS region, indicating the absence of a paternal allele (not shown)..$^{15}$ This is compatible with UPD15 mat. A submicroscopic deletion on the paternal chromosome 15q11 gives the same methylation pattern, but this was excluded by FISH using a cosmid probe from the SNRPN gene (fig 3C). A more detailed analysis of the parental origin of the chromosomes 15 was done using polymorphic markers distributed along this chromosome. Four of the six markers analysed were informative and showed that the patient had inherited two different 

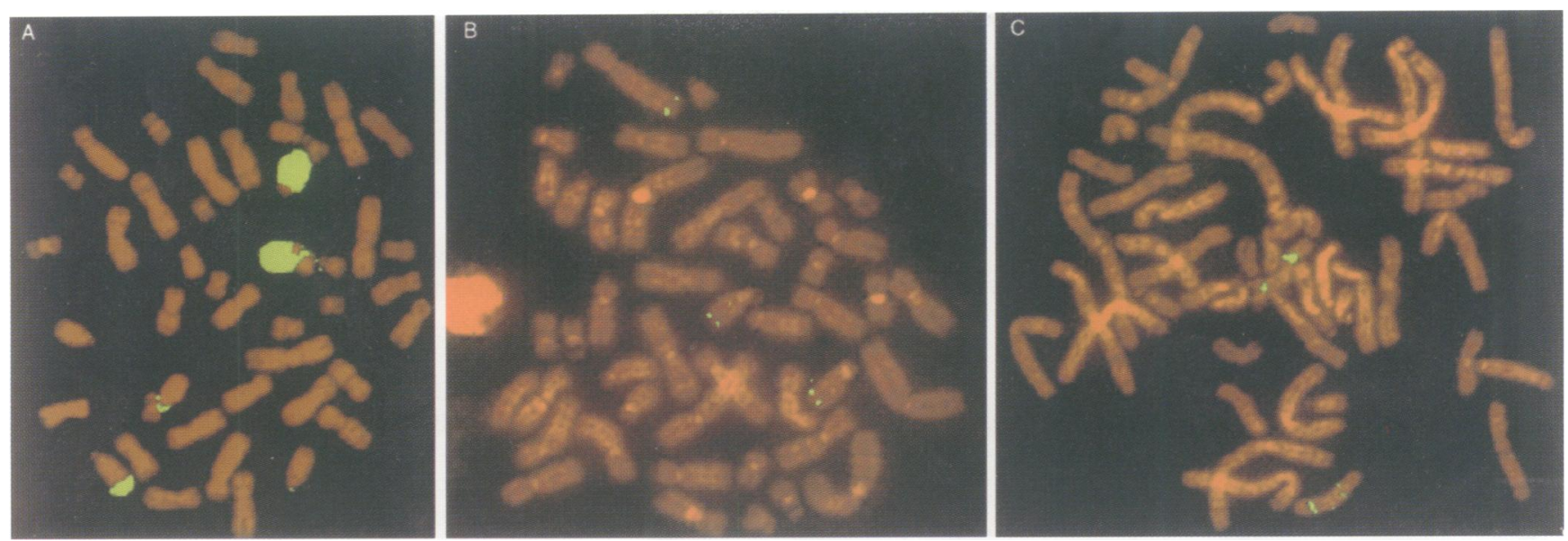

Figure 3 (A) FISH analysis using a chromosome 15 painting library. Note that the fragment translocated to chromosome 14qter originated from chromosome 15. (B) FISH analysis with cosmid probe FES (located on chromosome 15q26). Three distinct signals were present, two on the normal chromosomes 15 and one on the distal long arm of chromosome 4, at the site of the translocated fragment. (C) FISH analysis using the SNRPN/PML cocktail probe showed the presence of normal signals at $15 q 11$ and $15 q 22$ on both chromosomes, excluding a deletion involving the SNRPN gene. Moreover, the breakpoint on chromosome $15 q$ must be located between PML (15q22) and FES (15q26).

APW

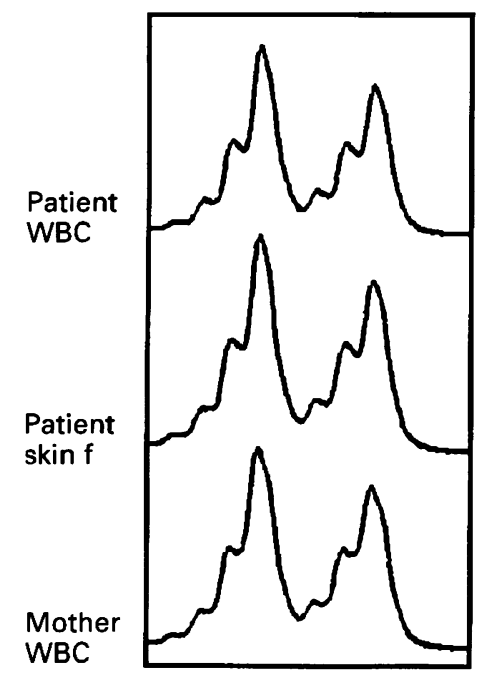

FES

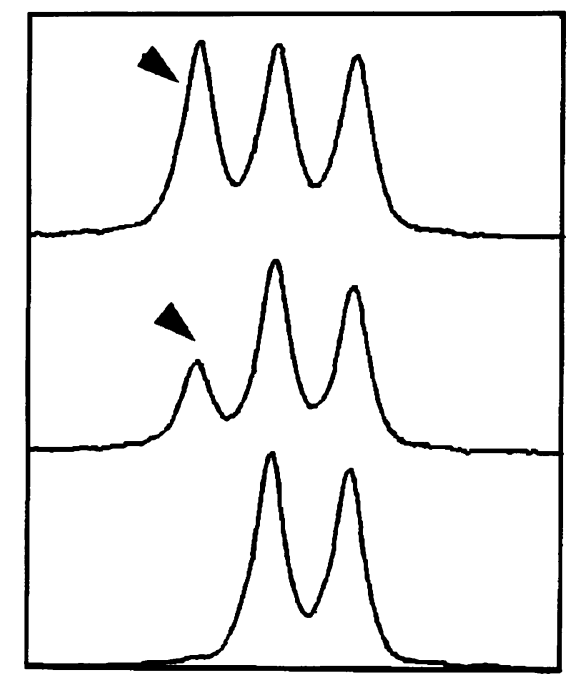

Figure 4 Analysis of microsatellite markers APW and FES. DNA from the patient's peripheral white blood cells (top row) and fibroblasts (middle row) and from maternal white blood cells (bottom row) was used for PCR amplification of the polymorphic repeats $A P W$ (dinucleotide repeat) and FES (tetranucleotide repeat). For FES, three alleles are observed for the patient, two of which are inherited from the mother, while the third must be of paternal origin (arrowhead). In fibroblasts, the amount of the paternal allele is reduced, reflecting the presence of mosaicism, with a normal $46, X X$ cell fibroblast line.

maternal chromosomes, which is fully compatible with the presence of maternal heterodisomy (fig 4, other results not shown). However, in the absence of paternal DNA, this could not be proven with absolute certainty.

The parental origin of the translocated chromosome $15 \mathrm{q}$ fragment was determined by means of polymorphic microsatellite markers in 15 q26 to 15 qter. Analysis of the polymorphic marker FES, located in 15q26, detected three different alleles, both in skin fibroblasts and in peripheral white blood cells (fig 4). One of the alleles was not present in the mother and therefore almost certainly represents the paternal allele. Similarly, for marker D15S107, also on distal 15q, an allele not present in the mother was found in the patient. This is in contrast to the markers proximal to $15 \mathrm{q} 24$, where no alleles absent in the mother could be found (results not shown). In conjunction with the cytogenetic and methylation studies, these findings are fully consistent with a paternal ori- gin of the translocated fragment of distal chromosome 15q.

Dosage analysis of the microsatellite markers FES and D15S107 showed that the paternal allele was present in the majority of white blood cells, whereas in skin fibroblasts a lower dose of the paternal allele was found compared to the maternal alleles (fig 4). This is in agreement with the cytogenetic findings, showing a partial trisomy for distal $15 \mathrm{q}$ in the majority of lymphocytes, but only in approximately $50 \%$ of fibroblasts (table 1).

INTERSTITIAL TELOMERE SEQUENCES AT THE BREAKPOINT JUNCTION SITES

The distal part of chromosome $15 \mathrm{q}$ was translocated onto three different chromosomes, $14 \mathrm{q}, 4 \mathrm{q}$, and $16 \mathrm{p}$. FISH, using a telomere probe, showed the presence of two signals on all normal chromosomes, including the two chromosomes 15 . On the chromosomes 14 and 4 , carrying the translocated $15 \mathrm{q}$ fragment, three signals were detected, two at the telomeres and one interstitial signal (fig 5). These interstitial signals coincided with the junction sites between the translocated distal chromosome $15 \mathrm{q}$ fragment and the translocation partners. No metaphases with a $16 \mathrm{p}+$ could be analysed.

\section{Discussion}

The patient reported here fulfils the diagnostic criteria of PWS according to Holm et al, ${ }^{12}$ with the presence of all main clinical features and seven minor criteria. By conventional cytogenetics, mosaicism consistent with a jumping translocation of distal $15 \mathrm{q}$ and resulting in a partial trisomy for distal $15 \mathrm{q}$ was detected in both lymphocytes and in fibroblasts. This rearrangement must have occurred de novo as the karyotypes of both parents were normal. Maternal uniparental heterodisomy for chromosome 15 was present, explaining the PWS phenotype. This was shown by methylation analysis of the imprinted region on chromosome $15 \mathrm{q} 11$ and further supported by the analysis of polymorphic microsatellite markers, distributed along chromosome 15 . On top of 


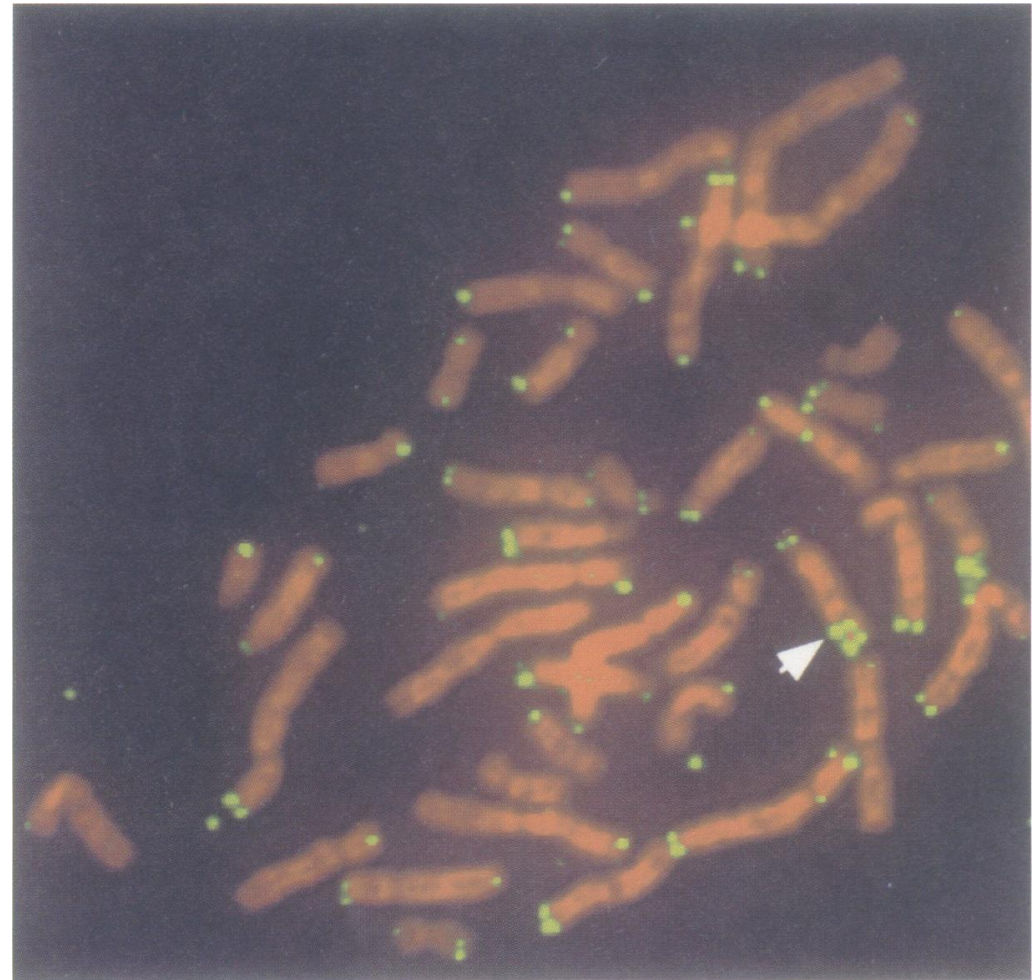

Figure 5 FISH analysis with a telomeric probe. Note that on the derivative chromosome 14 , with the extra distal 15q fragment, three signals are present, two at the ends of the chromosomes and one interstitial (indicated by arrow) at the translocation junction region.

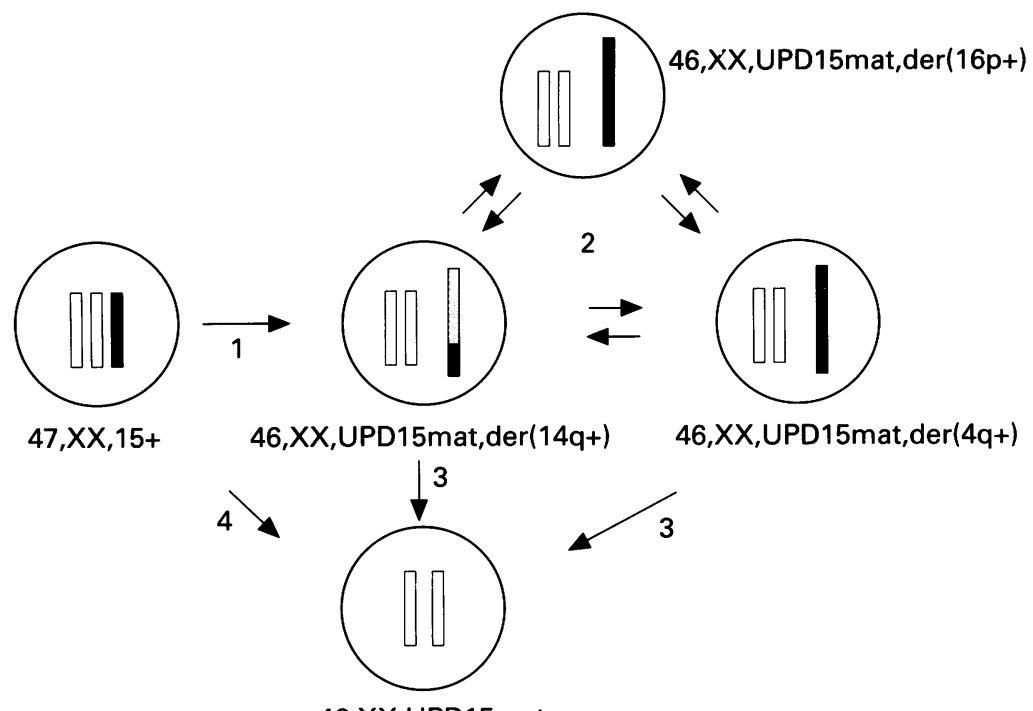

46,XX,UPD15mat of both intact chromosomes 15 , is fully compatible with a paternal origin of this translocated chromosomal fragment. The most likely mechanism explaining these findings is shown in fig 6 . Fertilisation by a normal male gamete of a disomic oocyte results in a zygote with trisomy 15 . These embryos are unviable, unless a postzygotic correction occurs. The loss of the paternal chromosome 15 results in a UPD15 mat. Trisomy rescue is a recognised mechanism leading to UPD. ${ }^{17}{ }^{18}$ Interestingly, whereas the correction usually involves the loss of the entire chromosome 15, a partial loss of chromosome 15 occurred in this patient. The distal chromosome 15q fragment was retained and translocated to other chromosomes, resulting in partial trisomy for distal 15q. To our knowledge, this is the first report showing that in trisomy rescue both UPD and partial trisomy can occur simultaneously.

The distal $15 \mathrm{q}$ fragment in this patient was present as a jumping translocation involving three different recipient partners, chromosomes $14 \mathrm{q}, 4 \mathrm{q}$, and $16 \mathrm{p}$. In addition, a normal $46, \mathrm{XX}$ cell line without the partial trisomy was found, an unprecedented finding in patients with a jumping translocation. ${ }^{311}$ This cell line also has a UPD15 mat, since in skin fibroblasts, where approximately $50 \%$ of the cells have a $46, \mathrm{XX}$ karyotype, an exclusively maternal methylation pattern was detected. The results of the microsatellite analysis are also fully consistent with this.

There are several different possible explanations for these observations. In a first possible mechanism, translocation of the distal $15 \mathrm{q}$ fragment to another chromosome coincides with the process of trisomy rescue (fig 6). During subsequent cell divisions, this fragment is then translocated to other chromosomes, that is, a real jumping process (fig 6). This mechanism has been proposed before. ${ }^{3}$ The euploid 46,XX cell line could be the result of the loss of the distal $15 \mathrm{q}$ chromosomal fragment during the jumping process (fig 6 , step 3). Alternatively, an independent trisomy rescue event might have occurred in a different cell line with the loss of an entire paternal chromosome 15 (fig 6, step 4) or after trisomy rescue, the distal chromosome $15 \mathrm{q}$ could initially remain as a free acentric chromosome fragment. During subsequent cell divisions and in different cells, the fragment could either be lost or translocated to different chromosomes.

In this patient, the telomeric regions of the translocation partners appeared cytogenetically intact. In addition, by means of FISH, interstitial telomeric sequences were shown at the breakpoint junctions. This would imply an initial translocation of the distal $15 q$ fragment to the telomere of one chromosome. The presence of interstitial telomeres might render these derivative chromosomes unstable and prone to breakage at this site and recombination with other telomeric sequences, as was suggested before. ${ }^{4}$ The jumping translocation process would then not represent a recurrent reciprocal translocation but rather a transposition of a chromosomal fragment from one telomere to another. Loss of the chromosome severe than usually observed in PWS. Paternal inheritance for alleles on chromosome 15 could only be shown for distal chromosome $15 \mathrm{q}$, and this, together with a maternal origin 
15qter fragment, leading to a euploid cell line, would occur upon chromosome breakage at the junction site without a simultaneous recombination with another telomere. Further experimental evidence to support this mechanism could be gained from long term cultures of clonal cell lines carrying a jumping translocation, and the finding of either a loss of the fragment or transposition to the telomeres of another chromosome.

Another proposed mechanism would require that the distal chromosome $15 \mathrm{q}$ acentric chromosome fragment remains stable during several sequential cell divisions before being lost or translocated to the telomeres of different chromosomes. It is difficult to envisage how a chromosomal fragment without a centromere and missing a telomere at one end could be stably retained during several cell divisions. Therefore, we favour the first mechanism.

Interestingly, besides one patient with a breakpoint in chromosome 17q23, all seven other reported patients with a telomeric translocation have a breakpoint in chromosome 15q11-13 and have the PWS phenotype..$^{4-6}$ Involvement of the same chromosomal region has led to the suggestion that in this chromosomal region specific DNA sequences must be present, with an affinity for recombination with telomeres. ${ }^{46}$ On the other hand, in the two other patients with a constitutional jumping translocation investigated so far, interstitial telomeric sequences were also found, as in the present case (case 1 of Park et al, ${ }^{5}$ case 3 of Rossi et $\left.a l^{4}\right)$. The detection of interstitial telomeres in the present patient with a breakpoint in 15q24 suggests that the jumping process could be related to the presence of interstitial telomeric sequences and not merely to the chromosomal region involved. Additional studies are needed and, more specifically, molecular cloning of the breakpoint region on $15 \mathrm{q} 24$ will be of particular interest in addressing this question.

In conclusion, the present observation is a further illustration of trisomy rescue leading to uniparental disomy and shows the presence of UPD in association with a partial trisomy. A similar observation has been made before in a patient with UPD16 mat and mosaic trisomy for distal $16 \mathrm{p} .{ }^{19}$ For the first time, interstitial telomere sequences were also found in a constitutional jumping translocation involving a chromosomal region outside 15q11-13. Therefore, we suggest that the jumping process is related to the presence of interstitial telomeres.
We thank Reinhilde Thoelen for expert technical help and Eric Schoenmakers for the FES probe. This work is supported by a krediet aan navorser, 1994, from the Nationaal Fonds voor Wetenschappelijk Onderzoek of Belgium. Peter Marynen is a onderzoeksdirecteur and Gert Matthijs and Joris Vermeesch are aangesteld navorsers of the Nationaal Fonds voor Wetenschappelijk Onderzoek, Belgium.

1 Ledbetter DH, Riccardi VM, Airhart SD, Strobel RJ, Keenan BS, Crawford JD. Deletions of chromosome 15 as a cause of the Prader-Willi syndrome. $N$ Engl $f \mathrm{Med}$ 1981;304:325-9.

2 Nicholls RD, Knoll JHM, Butler MG, Karam S, Lalande M. Genetic imprinting suggested by maternal heterodisomy in non-deletion Prader-Willi syndrome. Nature 1989;342. 281-5.

3 Rivera $H$, Zuffardi O, Gargantini L. Non-reciprocal and jumping translocations of 15q1-qter in Prader-Will syndrome. Am f Med Genet 1990;37:311-17.

4 Rossi E, Floridia G, Casali M, et al. Types, stability and phenotypic consequences of chromosome rearrangements leading to interstitial telomeric sequences. 7 Med Genet 1993;30:926-31.

5 Park VM, Gustashaw KM, Wathen TM. The presence of interstitial telomeric sequences in constitutional chromosome abnormalities. Am f Hum Genet 1992;50:914-23.

6 Reeve A, Norman A, Sinclair P, et al. True telomeric translocation in a baby with the Prader-Willi phenotype. $\operatorname{Am} \mathcal{F}$ Med Genet 1993;47:1-6.

7 Jauch A, Robson L, Smith A. Investigations with fluorescence in situ hybridisation (FISH) demonstrate loss of the telomeres on the reciprocal chromosome in three unbalanced translocations involving chromosome 15 in the Prader-Willi and Angelman syndromes. Hum Genet 1995; 96:345-9.

8 Robinson WP, Wagstaff J, Bernasconi F, et al. Uniparental disomy explains the occurrence of the Angelman or Prader-Willi syndrome in patients with an additional smal inv dup(15) chromosome. $\mathcal{F}$ Med Genet 1993;30:756-60.

9 Toth-Fejel S, Olson S, Gunter K, et al. The impact of imprinting: Prader-Willi syndrome resulting from chromosome translocation, recombination and nondisjunction. Am $\mathcal{F}$ Hum Genet 1996;58:1008-76.

10 Lejeune J, Maunoury C, Prieur M, Van den Akker J. Translocation sauteuse $(5 \mathrm{p} ; 15 \mathrm{q}),(8 \mathrm{q} ; 15 \mathrm{q}),(12 \mathrm{q} ; 15 \mathrm{q})$. Ann Genet (Paris) 1979;22:210-13.

11 Farrell SA, Winsor EJT, Markovic VD. Moving satellites and unstable chromosome translocations: clinical and cytogenetic implications. Am f Med Genet 1993;46:715-20.

12 Holm VA, Cassidy SB, Butler MG, et al. Prader-Willi syndrome: consensus diagnostic criteria. Pediatrics 1993 91:398-402.

13 Pinkel D, Straume T, Gray JW. Cytogenetic analysis using quantitative, high-sensitivity, fluoresence hybridisation. Proc Natl Acad Sci USA 1986;83:2934-8.

14 Vermeesch JR, De Meurichy W, Van Den Berghe $H$, Marynen P, Petit P. Differences in the distribution and nature of the interstitial telomeric (TTAGGG)n sequences in the chromosomes of the Giraffidae, okapi (Okapi Johnstoni), and giraffe (Giraffa camelopardalis): evidence for ancestral telomeres at the okapi polymorphic rob $(4 ; 26)$ fusion site. Cytogenet Cell Genet 1996;72:310-15.

15 Dittrich B, Robinson WP, Knoblauch H, et al. Molecular diagnosis of the Prader-Willi and Angelman syndromes by detection of parent-of-origin specific DNA methylation in 15q11-13. Hum Genet 1992;90:313-15.

16 Driscoll DJ, Waters MF, Williams CA, et al. A DNA methylation imprint, determined by the sex of the parent, distinguishes the Angelman and Prader-Willi syndromes. $G e-$ nomics 1992;3:917-24.

17 Cassidy SB, Lai LW, Erickson RP, et al. Trisomy 15 with loss of the paternal 15 as a cause of Prader-Willi syndrome due to maternal disomy. Am $\mathcal{H}$ Hum Genet 1992;51:701-8.

18 Engel E. La disomie uniparentale: revue des causes et conséquences en clinique humaine. Ann Genet (Paris) 1995;38:113-16.

19 Schinzel A, Kotzot D, Brecevic L. An unusual consequence of maternal uniparental disomy 16. Genet Counsel 1996;7:87. 